\title{
Middle lobe syndrome due to calcified adenopathy
}

\author{
Akram Arab MD, Nha Voduc MD, Terence Moyana MD, Sunita Mulpuru MD
}

\begin{abstract}
A 43-year-old nonsmoking man presented with a two-year history lof recurrent respiratory infections. He had previously been given a clinical diagnosis of asthma. A review of systems was negative for aspiration, constitutional symptoms, occupational exposures and risk factors for tuberculosis and HIV. Spirometry demonstrated reduced forced expiratory volume in $1 \mathrm{~s}$ ( $78 \%$ predicted) without airflow limitation, and a methacholine challenge test was negative for asthma. Immunoglobulin levels and complete blood counts were normal. A chest radiograph (Figure 1) was abnormal, prompting a computed tomography (CT) scan of the thorax (Figures 2A and 2B).

Bronchoscopic visualization of the right middle lobe bronchus revealed complete obstruction by inflamed bronchial mucosa and external compression. The hilar mass was identified and biopsied under endobronchial ultrasound guidance. A pathological review of
\end{abstract}
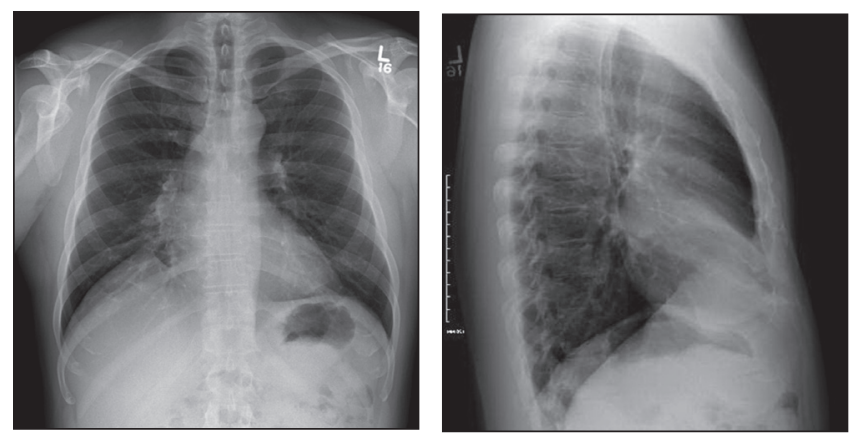

Figure 1) Posterior-anterior chest radiograph showing right side volume loss with elevated right hemi diaphragm, prominence of the right hilum, and the silhouette sign over the right heart border. The lateral radiograph (right panel) confirms the right middle lobe atelectasis based on the triangular density in the anterior chest overlaying the base of the cardiac shadow. The horizontal fissure is displaced inferiorly
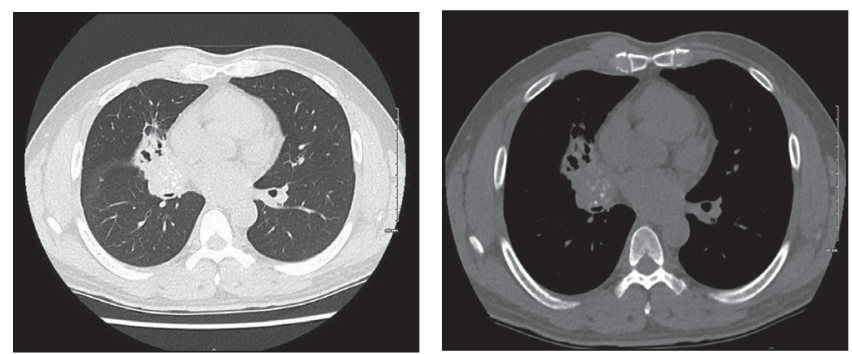

Figure 2) Computed tomography of the thorax with lung field and mediastinal views. Lung views demonstrate narrowing of the right lower lobe bronchus and obliteration of the right middle lobe bronchus opening due to a hilar mass. Accompanying bronchiectasis presents in the right middle lobe. Mediastinal views demonstrate the punctate calcifications within this mass. No other adenopathy or suspicious pulmonary nodules were identified biopsy specimens was negative for malignancy and revealed a mixed population of benign lymphocytes with no granulomas (Figures 3A and $3 \mathrm{~B})$. No mycobacteria were cultured.

The patient was diagnosed with middle lobe syndrome (MLS) secondary to airway compression from an enlarged, partly calcified hilar lymph node, likely secondary to previous Histoplasma exposure given his residence in an endemic area. He was referred to thoracic surgery and scheduled for right middle lobectomy.
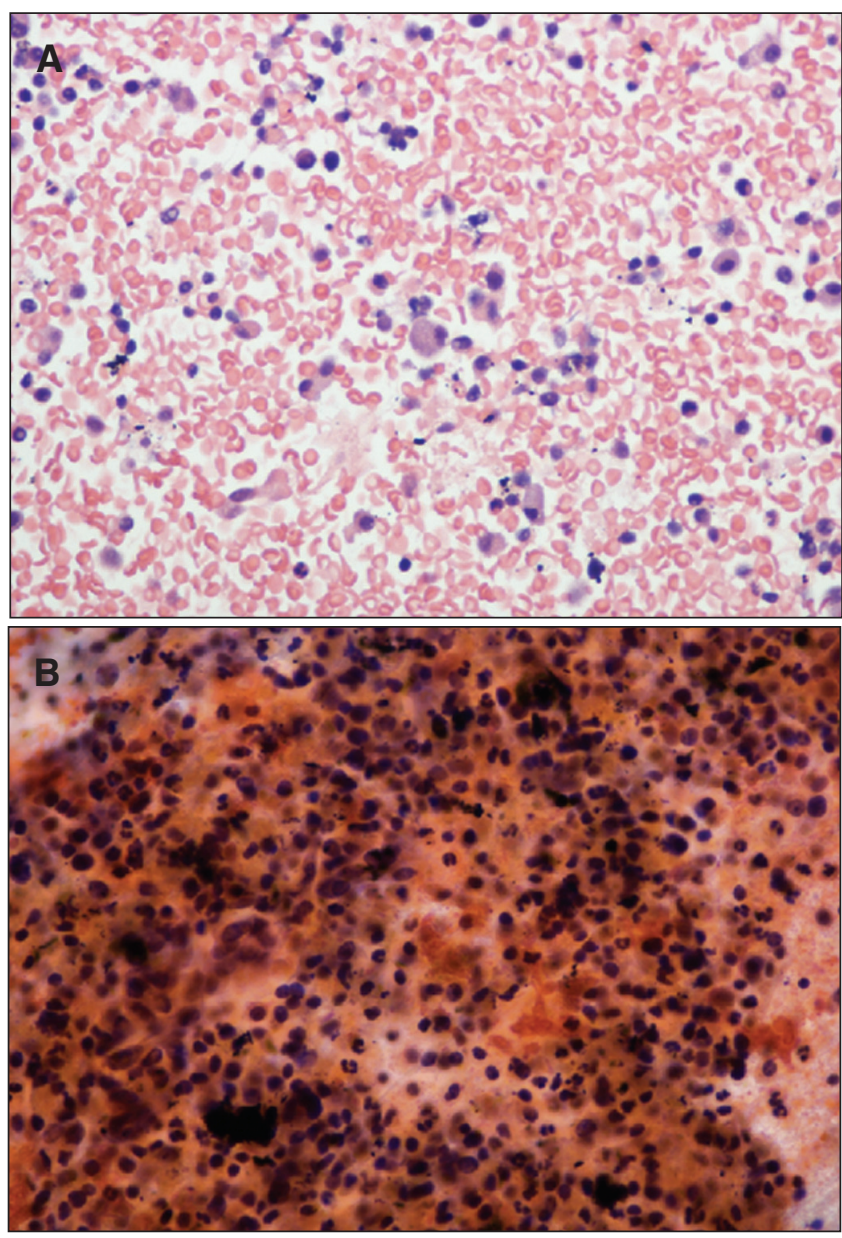

Figure 3) A Cell block preparation showing small and large lymphocytes, plasma cells and macrophages. Note the absence of malignant cells (hematoxylineosin stain, original magnification $\times 40$ ). B Cellular area of the cytology smear showing a polymorphous leucocytic infiltrate that is characteristic of a reactive lymph node (Papanicolaou stain, original magnification $\times 40$ )

Department of Medicine, University of Ottawa, Ottawa, Ontario

Correspondence: Dr Sunita Mulpuru, Department of Medicine, Division of Respirology, The Ottawa Hospital, General Campus, Box 211,

501 Smyth Road, Ottawa, Ontario K1H 8L6. Telephone 613-737-8899 ext 73994, fax 613-737-8537,

e-mailsunitamulpuru@hotmail.com 


\section{KEY LEARNING POINTS}

- MLS is a rare condition, first described in 1955 (1), and is characterized by recurrent or chronic collapse of the right middle lobe or lingula.

- A history of recurrent infections is a common presentation of MLS (2).

- Common causes of MLS due to extrinsic compression are granulomatous infections (eg, histoplasmosis, mycobacteria), sarcoidosis and lymph node metastases (2).

- Clinicians should consider thoracic surgery referral in cases for which recurrent infection is refractory to antibiotic therapies and obstruction of the right middle lobe is proven (3).

- There is limited evidence in adults, but operative complications and morbidity appear to be minimal (3).

\section{REFERENCES}

1. Effler DB, Ervin JR. The middle lobe syndrome; a review of the anatomic and clinical features. Am Rev Tuberc 1955;71:775-84.

2. Gudbjartsson T, Gudmundsson G. Middle lobe syndrome: A review of clinicopathological features, diagnosis and treatment. Respiration 2012;84:80-6.

3. Einarsson JT, Einarsson JG, Isaksson H, et al. Middle lobe syndrome: A nationwide study on clinicopathological features and surgical treatment. Clin Respir J 2009;3:77-81.

The 'Images in Respiratory Medicine' section of the Canadian Respiratory Journal aims to highlight the importance of visual interpretation, whether physiological, radiological, bronchoscopic, surgical/thorascopic or histological, in the diagnosis of chest diseases. Submissions should exemplify a classic, particularly dramatic or intriguing presentation of a disease while offering an important educational message to the reader (insightful diagnostic pearls or differential diagnosis, etc). This section is not intended to be a vehicle for publication of case reports (see the Clinical-Pathologic Conferences for case-based leaning series). 


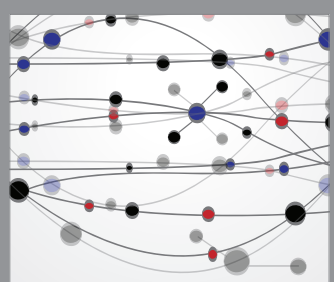

The Scientific World Journal
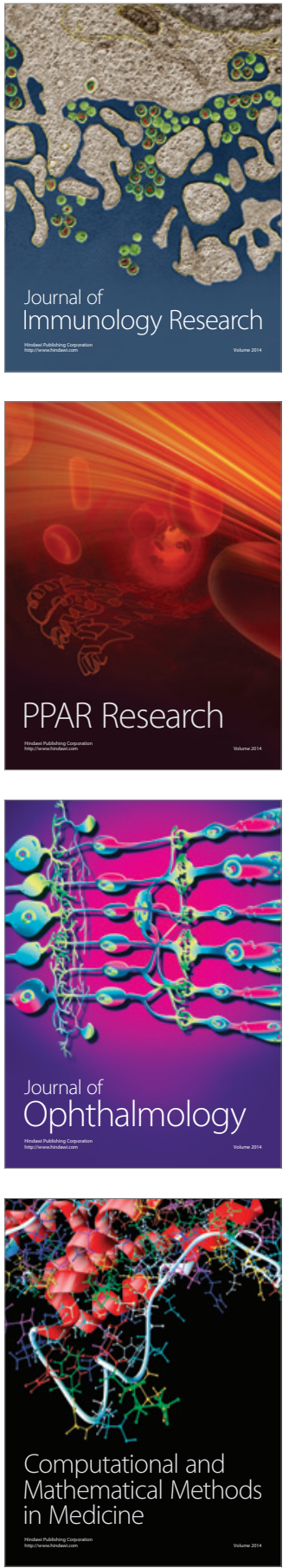



Gastroenterology Research and Practice

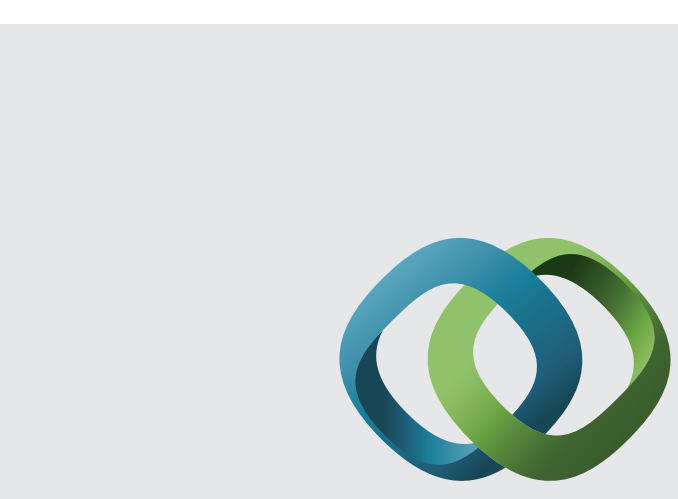

\section{Hindawi}

Submit your manuscripts at

http://www.hindawi.com
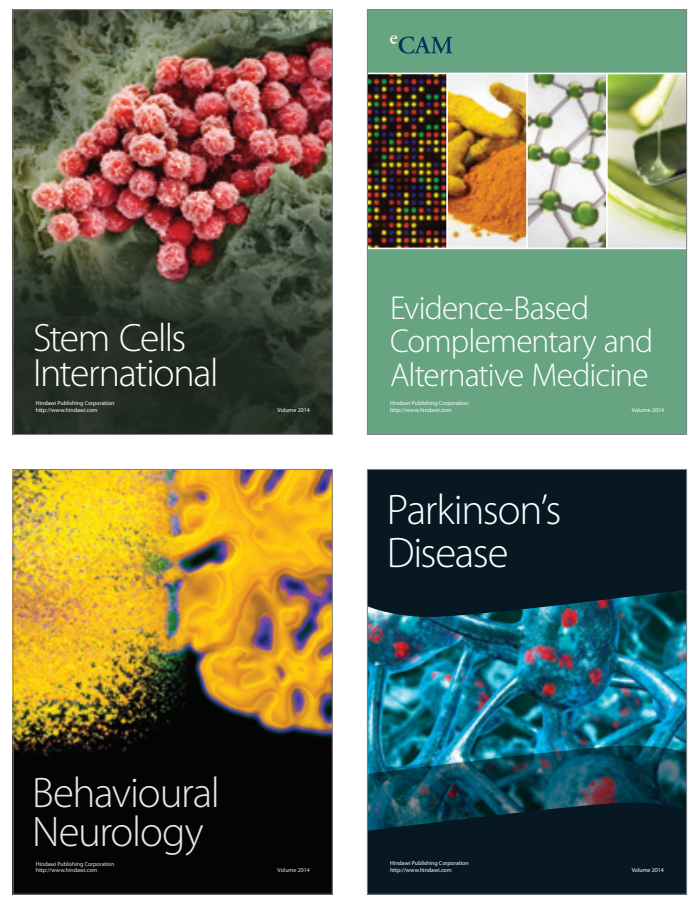
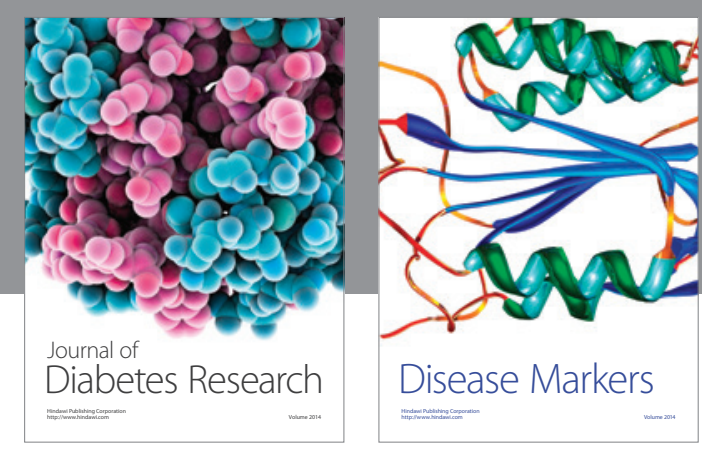

Disease Markers
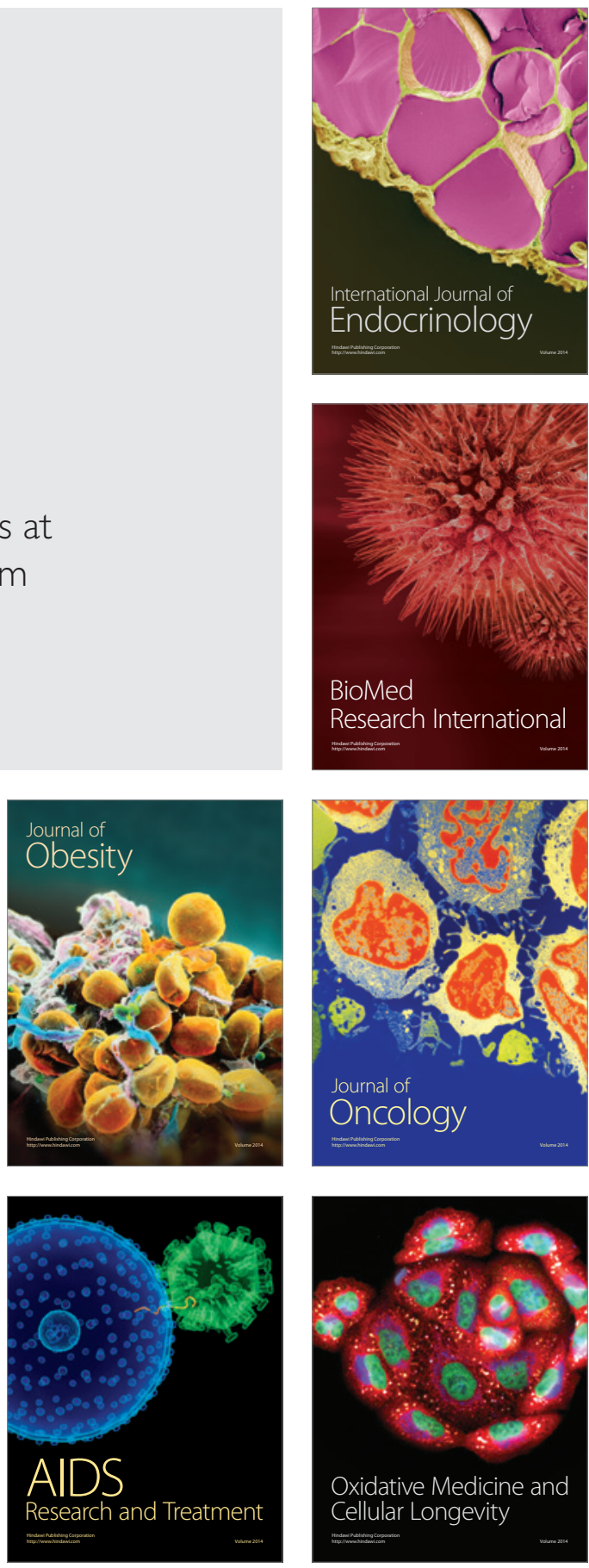